\title{
An Open-Source Algorithm for Standardized Bullseye Visualization of High- Resolution Cardiac Ventricular Data: UNISYS
}

\author{
Job Stoks $^{1,2,3}$, Uyen C Nguyen ${ }^{4}$, Ralf Peeters ${ }^{2}$, Paul GA Volders ${ }^{1}$, Matthijs JM Cluitmans ${ }^{1}$ \\ ${ }^{1}$ Department of Cardiology, Maastricht University, Maastricht, the Netherlands \\ ${ }^{2}$ Data Science and Knowledge Engineering, Maastricht University, Maastricht, the Netherlands \\ ${ }^{3}$ BIOMED, Hasselt University, Hasselt, Belgium \\ ${ }^{4}$ Department of Physiology, Maastricht University, Maastricht, the Netherlands
}

\begin{abstract}
Standardized visualization of electro- or mechanoanatomical data allows easy inter- and intra-patient comparison. For this purpose, we developed the opensource and freely available UNISYS (Universal Ventricular Bullseye Visualization) software. A patientspecific mesh of the ventricular anatomy typically consists of a certain number of vertices and their associated values. Based on a limited amount of user inputs, the algorithm transforms these $3 D$ single-layer coordinates to a circular $2 D$ disk ('bullseye') through a number of translations and rotations, and interpolates them to achieve a continuous standardized visualization. The algorithm shows a high degree of bidirectionality and a robust spatial preservation of points of interest.
\end{abstract}

\section{Introduction}

Processing electro- or mechano-anatomical results from cardiac ventricles for scientific purposes in a structured manner can be challenging. For example, displaying the full three-dimensional electrocardiographic imaging (ECGI) observation of a single subject currently requires three to four different views to cover all relevant cardiac structures. Moreover, comparing results of different subjects is difficult, due to inter-individual differences in anatomy. Providing a clear, unified graphical representation of data by standardization, whilst still considering inter-individual anatomical aspects, could aid researchers in gaining more insight from complex data. Such methods exist, but are either not open-source, can only visualize the left ventricle (LV) [1], require complex calculations [1-2], are solely focused on imaging anatomy [2] or have limited visualization options. We developed UNISYS (Universal Ventrịcular Bullseye Visualization): an open-source algorithm to process the geometry of cardiac ventricular data and to display them uniformly in a 2D high-resolution circular representation (bullseye plot), to overcome these disadvantages. The algorithm provides a semi-automatic approach programmed in MATLAB $®$ and is suitable for invasive and non-invasive electro- or mechanical-anatomical mapping data of the ventricles (either whole epicardium or LV endocardium only). The algorithm works with any kind of ventricular data, as long as they are represented in a single layer, e.g. endocardial or epicardial. The current paper illustrates its application through epicardial ECGI data incorporating both the LV and the right ventricle (RV).

\section{Methods}

The geometry is built on the assumption that the ventricles are defined by vertices, and each vertex contains an associated value (e.g. activation time, repolarization time, voltage etc.) to display. Faces connecting these vertices (e.g. triangles) are not required. The algorithm, which is written in MATLAB ${ }^{8} \mathrm{R} 2017 \mathrm{~b}$, firstly requires a number of user inputs. Secondly, through a series of translations and rotations, the ventricles are rotated upright, parallel to heart axis, with the apex pointing downwards. Thirdly, the ventricles are morphed to a coneshape. Lastly, the cone shape is flattened to a 2D surface, to achieve the UNISYS bullseye visualization. The key steps to this pipeline are shown in Figure 1 and discussed below.

\subsection{User inputs}

Firstly, the user is asked to denote the three vertices on the heart to denote a plane through the septum. The first point should be denoted on the apical side of the heart, and the remaining two points on the basal side. The plane crossing the three points is considered the septum (see Figure 1B), and the user then defines which of the two ventricles is the left ventricle. Secondly, if the basal nodes contain any values, the user is asked to denote three points which separate the basal nodes (representing the annulus fibrosus and intermediary tissue) from the rest of the ventricles. These basal nodes will not be represented in the final bullseye as they typically do not contain actual data. 

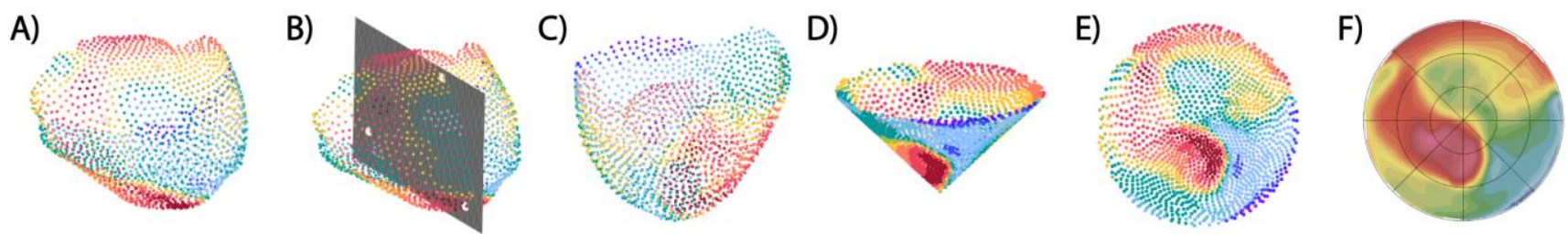

Figure 1: UNISYS pipeline. A) Input: vertices of heart, each with an associated value (color-coded here). B) The algorithm requires the user to denote three coordinates indicating the septum. C) After obtaining the user input, the heart is translated and rotated upright. D) The heart is transformed to a cone shape, with increasing radius from apex to base. E) The cone and its associated values are transformed onto a ciruclar 2D disk. F) Associated values of remaining vertices are interpolated to render a continuous representation.

Through these inputs, the algorithm is provided with the current orientation of the ventricles.

\subsection{Translation and rotation}

After obtaining the current orientation of the ventricles, any vertices not containing an associated value are deleted. Subsequently, the vertices are translated and rotated upright (parallel to the z-axis), see Figure 1C. In order to achieve this, firstly, the ventricles are translated so that the apex is moved to the origin. Secondly, the ventricles are rotated through several rotation matrices by determining the angle of the heart axis with the $\mathrm{x}, \mathrm{y}$ and $\mathrm{z}$-axis. Lastly, the ventricles are translated once more to make sure the lowest $\mathrm{z}$-coordinate equals zero.

\subsection{Cone shape}

Next, the ventricles are morphed to the shape of a cone, with increasing radius when moving from the apex to the base, see Figure 1D. To this end, cartesian coordinates of the upright ventricles are converted to polar coordinates. Polar coordinates consist of three elements: the angle with the origin in the xy-plane $(\theta)$, the distance to the $z$-axis $(\rho)$ and the distance to the xy-plane (z), see Figure 2. The 3D heart is converted to a cone shape (see Figure 1D) by first computing an $\varepsilon$ for each vertex: $\varepsilon=\sqrt{\mathrm{z}^{2}+\rho^{2}}$. Subsequently, all z- and $\rho$-coordinates are both replaced by the associated $\varepsilon$-value, to achieve the cone shape. The $\varepsilon$ of the outer (basal) ring is not equal throughout the ventricles. Consequently, a normalization of $\varepsilon$ for each $\theta$ is performed.

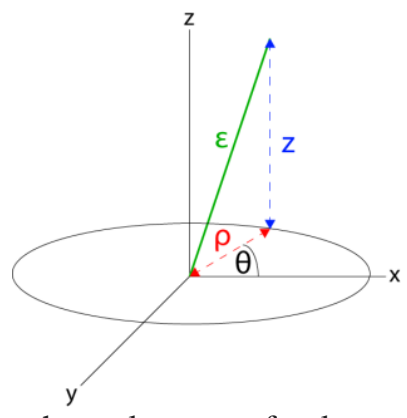

Figure 2: The three elements of polar coordinates: the angle with the origin in the xy-plane $(\theta)$, the distance to the $\mathrm{z}$-axis $(\rho)$ and the distance to the $\mathrm{xy}$-plane $(\mathrm{z})$ $\varepsilon$ is normalized for its maximum value as determined by a moving average-filter within a window of 50 samples (i.e., the filter moves over the outer circle defined by the maximum available epsilon value). Lastly, the polar coordinates of the heart (in which both $\mathrm{z}$ and $\rho$ are replaced by $\varepsilon$ ) are converted back to cartesian coordinates.

\subsection{UNISYS representation}

To achieve a final bullseye visualization, firstly the $\mathrm{z}$ coordinate of the resulting cone is disregarded, yielding a circular 2D disk (see Figure 1E). Thereafter, associated values of remaining vertices are projected on a standardized circular grid of 36,000 points. Lastly, these points are interpolated to a continuous representation, see Figure 1F.

\subsection{Bidirectionality and interpretability}

After transforming the ventricular data to the UNISYS representation, the bidirectionality and interpretability of the method were addressed. This was done in multiple ways, in four human hearts of patients with varying age and sex (see Table 1). 1) For each heart, one depolarization time pattern and one repolarization time pattern from ECGI were transformed to a UNISYS representation, see Figure 3. Afterwards, these values were re-transformed back to the 3D heart, by selecting the values of the grid point closest to each original vertex. The differences between the original data and the re-transformed data were investigated

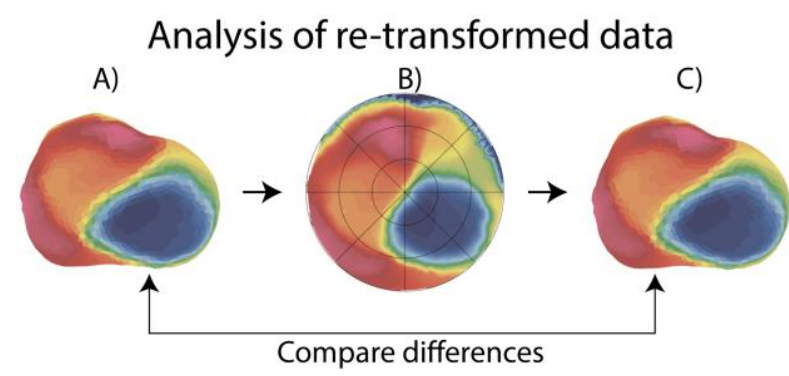

Figure 3: Analysis of re-transformed data. Original 3D data (A) were transformed to 2D UNISYS representation $(\mathrm{B})$, and back again $(\mathrm{C})$ ). Differences were quantified and are shown in Figure 4. Repolarization time data of subject 2 are shown here. 


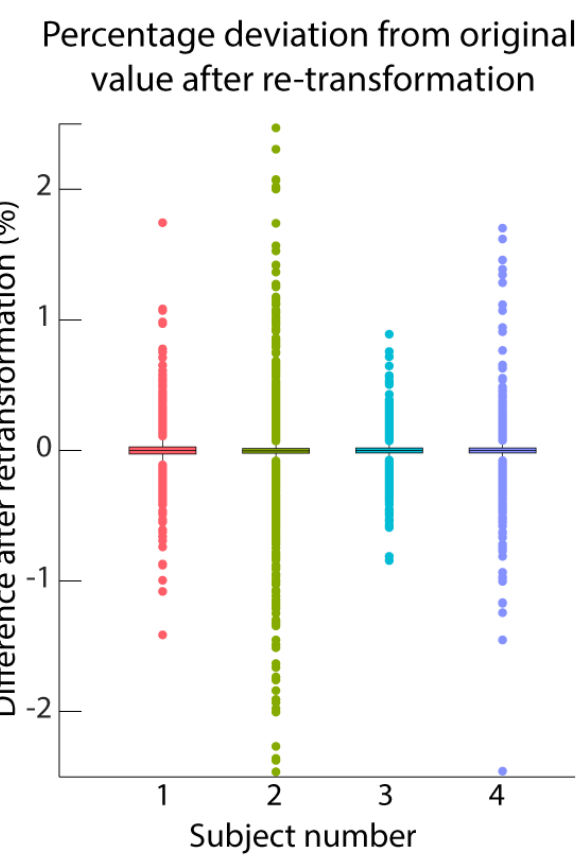

Figure 4: Boxplots of differences between original data on 3D heart and the re-transformed data. For each subject, one depolarization and one repolarization pattern from ECGI were used. Y-axis cropped at 2.5\% for visualization purposes.

and quantified. 2) The original user-inputs containing information about the user-defined septum were plotted on the UNISYS visualization, to assess the conservation of spatial relationship between both visualizations. 3) The UNISYS visualization was divided into 24 segments. This division of segments was converted back onto the original hearts, and visualized, to assess the structural properties and size (in number of vertices) of each of the segments.

\section{Results}

UNISYS was successfully employed in four human hearts containing ECGI data on depolarization and repolarization.

\subsection{Bidirectionality}

To assess bidirectionality of UNISYS, one depolarization pattern and one repolarization pattern were visualized on each of the four 3D hearts, see Figure 3 for an example (patient 2, repolarization). Figure 4 shows differences between the original and the re-transformed data, for each subject. Mean absolute deviation was $0.07 \%$. $99^{\text {th }}$ percentile of all absolute deviations was $0.9 \%$. Maximum absolute deviation over all 8 patterns was $4.8 \%$.
Table 1. Subject characteristics

\begin{tabular}{ccc}
\hline Subject & Age $(\mathrm{y})$ & Sex \\
\hline 1 & 67 & $\mathrm{~F}$ \\
2 & 53 & $\mathrm{~F}$ \\
3 & 50 & $\mathrm{M}$ \\
4 & 48 & $\mathrm{M}$ \\
\hline
\end{tabular}

\subsection{User-defined septum and 24 segments}

After achieving UNISYS visualization, the original user-defined septum coordinates were visualized on the UNISYS bullseye. Moreover, the interpretability of the UNISYS representation is shown by dividing the heart into 24 segments. Results are shown in Figure 5. Each segment in the UNISYS representation contains the percentage of vertices in that segment.

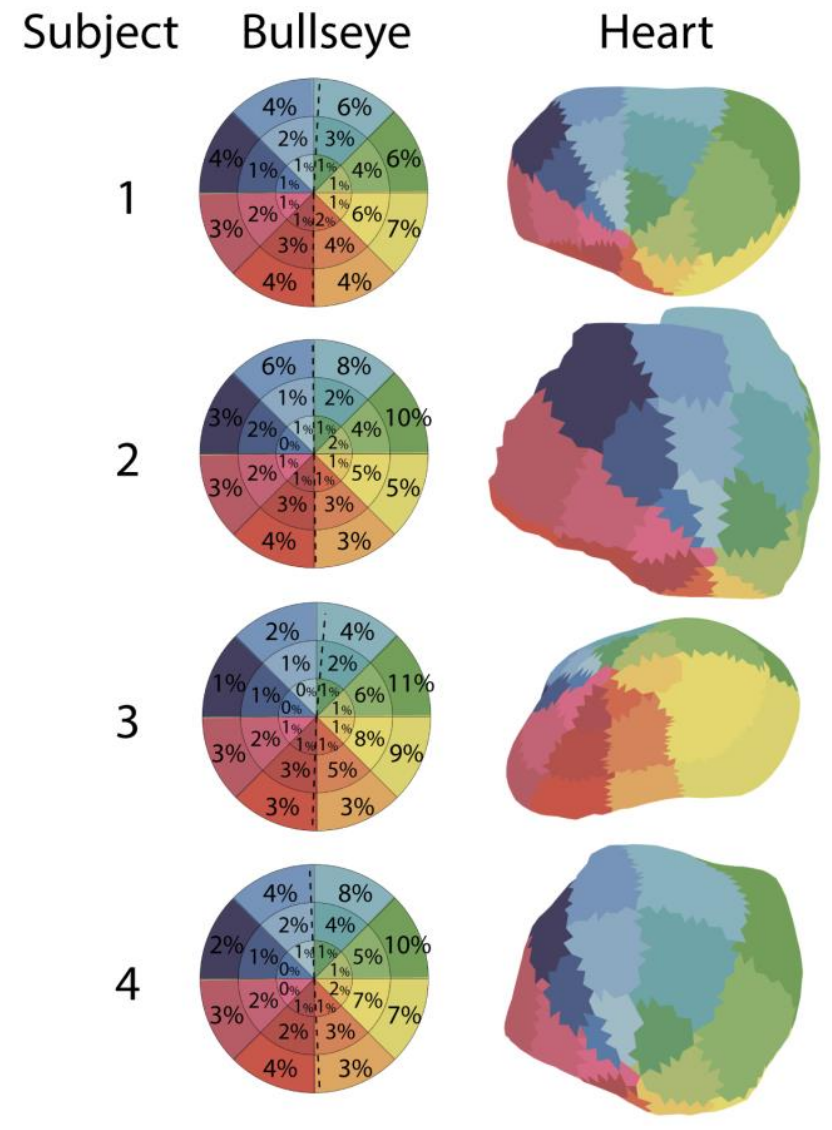

Figure 5: hearts divided into 24 segments. Left: UNISYS bullseye representation. Original user-defined septum coordinates are shown by the dashed line. Each segment contains the percentage of vertices on the heart corresponding with that segment. Right: 3D heart. 


\section{Discussion}

In this study, we developed a standardized, open-source method to visualize single-layer $3 \mathrm{D}$ ventricular data in a circular bullseye representation, named UNISYS. Any kind of data can be visualized, such as electrical, mechanical, anatomical or a combination of either of these. Also, data of a single ventricle can also be visualized with UNISYS in the same manner. This bullseye visualization allows for easy visual comparison of inter- and intrapatient observations.

As shown in Figures 3 and 4, the method shows high consistency in terms of bidirectionality. When transforming 3D data to UNISYS representation and back to 3D again, differences are very small. Some of these differences may arise from the fact that the 3D-to-UNISYS transformation was done by means of interpolation, while the UNISYS-to-3D transformation was done by means of nearest-neighbors.

The high degree of agreement between 3D and UNISYS visualization is also visible in Figure 5: the originally denoted user-defined septum coordinates (dashed line) show good agreement with the UNISYS-transformed septum coordinates (line dividing the left vs right side of the circle). However, the location of the user-defined septum does influence the surface area of each of the 24 segments, and thus the density of data in the UNISYS representation. The more equal both ventricles are in size, the more equal the density of data is for each of the 24 segments, which is most true in subject 1 . Conversely, as visible in subject 3 in whom the septum was defined more laterally to the right, the percentage of vertices in the outer ring can vary from $1 \%$ (RV lateral wall) to $11 \%$ (LV lateral wall) in a single subject. If the user-defined septum is placed more laterally, the density of data will increase in the smallest ventricle (or conversely: the size of each segment will decrease), as can be seen in subject 4, Figure 5). Users interpreting this bullseye visualization should realize that data density may differ and can be made aware of this with separate density visualizations when relevant.

Several other methods to transform 3D ventricular data to a standardized form are known. For example, the method by De Craene et al. to visualize a standardized anatomy requires the calculation of a Laplacian to convert the LV into the AHA 17-segment representation.[1] In contrast, our method can also be used to visualize both the LV and RV. The open-source method by Paun et al. [2] produces a patient-independent representation of the detailed cardiac anatomy onto a circular disk. Their method can also separate LV and RV. Conversely, our method focuses primarily on displaying electro-or mechano-anatomical results. Lastly, the universal ventricular coordinate system (UVC) by Bayer et al. also maps position within the ventricles of hearts to a generic frame of reference based on Laplacian solutions [3]. Even though their method was not used to project ventricular data on a standardized circle, it could be suitable to do so.

Even though not shown in this paper, UNISYS supports many optional visualization possibilities. For example, users can select their own (customized) colors of interest and bin width and color axis limits.

Lastly, a future release of UNISYS will support the AHA 17-segment bullseye plot. The RV and LV could be separated to create their own separate standardized visualizations. Moreover, if possible, UVC can be extracted from the UNISYS representation in a future release.

\section{Conclusion}

UNISYS is a standardized, open-source method to visualize single-layer 3D ventricular data in a circular bullseye representation for improved visual comparison of complex three-dimensional inter- and intra-patient observations. Even though interpretability could vary slightly based on individual patient anatomy (inherent to any standardized visualization), the method is highly consistent in terms of bidirectionality and spatial conservation of points of interest. UNISYS is freely available through the Consortium for ECG Imaging (CEI) through www.ecg-imaging.org.

\section{Disclosure of conflicts of interest}

Matthijs Cluitmans is part-time employed by Philips Research.

\section{Acknowledgments}

The authors would like to thank Mark Potse en Francesco Maffessanti for their ideas and technical support in the early stages of developing the current method.

\section{References}

[1] M. De Craene et al., "Temporal diffeomorphic free form deformation (TDFFD) applied to motion and deformation quantification of tagged MRI sequences," Lect. Notes Comput. Sci. (including Subser. Lect. Notes Artif. Intell. Lect. Notes Bioinformatics), vol. 7085 LNCS, pp. 68-77, 2012.

[2] B. Paun, B. Bijnens, T. Iles, P. A. Iaizzo, and C. Butakoff, "Patient independent representation of the detailed cardiac ventricular anatomy," Med. Image Anal., vol. 35, pp. 270-287, 2017.

[3] J. Bayer et al., "Universal ventricular coordinates: a generic framework for describing position within the heart and transferring data," Med. Image Anal., vol. 45, pp. 83-93, 2018. 